\title{
Multiadaptive Bionic Wavelet Transform: Application to ECG Denoising and Baseline Wandering Reduction
}

\author{
Omid Sayadi and Mohammad B. Shamsollahi \\ Biomedical Signal and Image Processing Laboratory (BiSIPL), School of Electrical Engineering, \\ Sharif University of Technology, P.O. Box 11365-9363, Tehran, Iran
}

Received 7 May 2006; Revised 22 October 2006; Accepted 11 January 2007

Recommended by Maurice Cohen

We present a new modified wavelet transform, called the multiadaptive bionic wavelet transform (MABWT), that can be applied to ECG signals in order to remove noise from them under a wide range of variations for noise. By using the definition of bionic wavelet transform and adaptively determining both the center frequency of each scale together with the $T$-function, the problem of desired signal decomposition is solved. Applying a new proposed thresholding rule works successfully in denoising the ECG. Moreover by using the multiadaptation scheme, lowpass noisy interference effects on the baseline of ECG will be removed as a direct task. The method was extensively clinically tested with real and simulated ECG signals which showed high performance of noise reduction, comparable to those of wavelet transform (WT). Quantitative evaluation of the proposed algorithm shows that the average SNR improvement of MABWT is $1.82 \mathrm{~dB}$ more than the WT-based results, for the best case. Also the procedure has largely proved advantageous over wavelet-based methods for baseline wandering cancellation, including both DC components and baseline drifts.

Copyright (c) 2007 O. Sayadi and M. B. Shamsollahi. This is an open access article distributed under the Creative Commons Attribution License, which permits unrestricted use, distribution, and reproduction in any medium, provided the original work is properly cited.

\section{INTRODUCTION}

The heart is a hollow muscular organ which through a coordinated muscle contraction generates the force to circulate blood throughout the body. Each beat of our heart is triggered by an electrical impulse from special sinus node cells in the atrium. The electrical impulse travels to other parts of the heart and causes the heart to contract. An electrocardiogram (ECG) records these electrical signals. A normal ECG describes the electrical activity in the heart, and can be decomposed in characteristic components, named the P, Q, R, $\mathrm{S}$, and $\mathrm{T}$ waves. Each of these components has its own typical form and behavior and each heart beat traces the familiar morphology labeled by these peaks and troughs as shown in Figure 1.

When an electrocardiogram is recorded, it would be contaminated with many kinds of noise [1], such as the following.

(i) Baseline wandering, which can be modeled by low pass noise.

(ii) 50 or $60 \mathrm{~Hz}$ power-line interference. (iii) Electromyogram (EMG), which is an electric signal caused by the muscle motion during effort test.

(iv) Motion artifact, which comes from the variation of electrode-skin contact impedance produced by electrode movement during effort test.

Since ECG is mostly contaminated with noise, extraction of pure cardiological indices from noisy measurements has been one of the major concerns of biomedical signal processing and needs reliable signal processing techniques to preserve the diagnostic information of the recorded signal. For example, the S-T segment in the ECG signal is used for diagnosing ischemia, myocardial infarction and indicating an imbalance of myocardial oxygen supply. The aim of this paper is to remove common noise caused by motion artifact, Figure 2(b), and also baseline wandering, Figure 2(c).

Techniques have been proposed to extract the ECG components contaminated with the background noise and allow the measurement of subtle features in the ECG signal. One of the common approaches is the adaptive filters architecture which has been used for the noise cancellation of ECGs containing baseline wandering, power line interference, EMG 


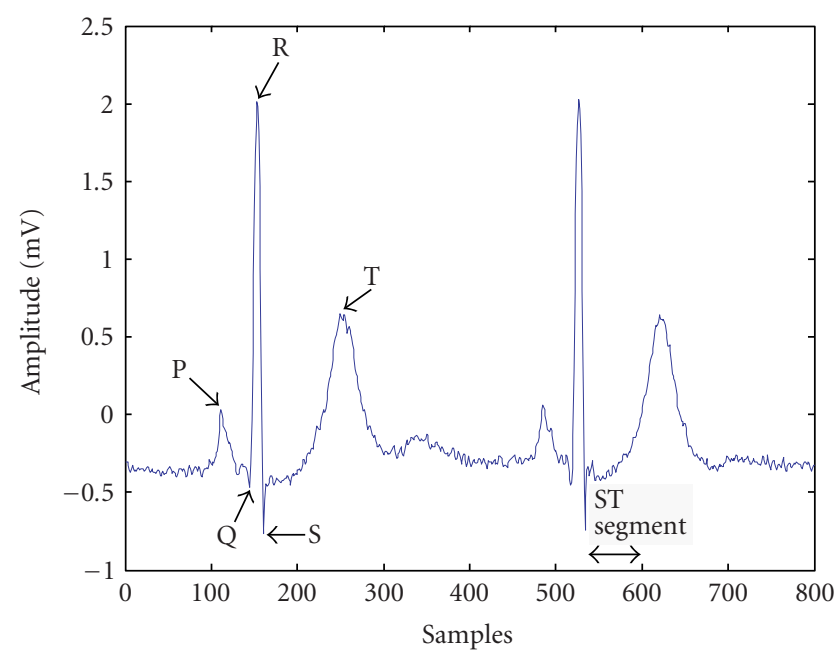

FIgUre 1: A typical human's ECG signal.

noise, and motion artifacts $[2,3]$. Statistical techniques such as principal component analysis [4], independent component analysis [5, 6], and neural networks [7] have also been used to extract a noise-free signal from the noisy ECG. Over the past several years, wavelet transform (WT) methods have also received great deal of attention for denoising of signals having multiresolution characteristics such as the electrocardiogram [8-12].

Besides the above algorithms, baseline wandering removal has been addressed in the literature individually. Baseline estimation using cubic spline [13], baseline construction by linearly interpolating between preknown isoelectric levels estimated from PR intervals [14], linear filtering [15], and the use of wavelet packets [16] are major approaches in this field.

Among transform-based methods, bionic wavelet transform (BWT), introduced by Yao and Zhang [17], is mainly developed and being optimized by the human biosystem and has showed promising results in speech processing. The term "bionic" goes back to the fact that BWT was originally inspired by a biological mechanism which is related to the human's biosystem. This idea motivated us to apply the BWT for processing the electrocardiograms. In this paper we attempted to apply BWT with new modifications to be properly adjusted for ECG processing, especially for denoising applications. The new proposed algorithm employs a multiadaptation scheme and leaves the multiadaptive bionic wavelet transform as a novel ECG analyzer.

The paper is organized as follows. Section 2 provides theoretical background on the definition of the bionic wavelet transform. In Section 3 BWT is optimized for ECG signal analysis with modifying the multiadaptation scheme. Section 4 focuses on denoising and investigates the multiadaptive bionic wavelet transform to be applied to ECG for denoising and baseline wandering cancellation. Finally, the simulation results are provided in Section 5 followed by discussion and conclusions coming in Section 6.

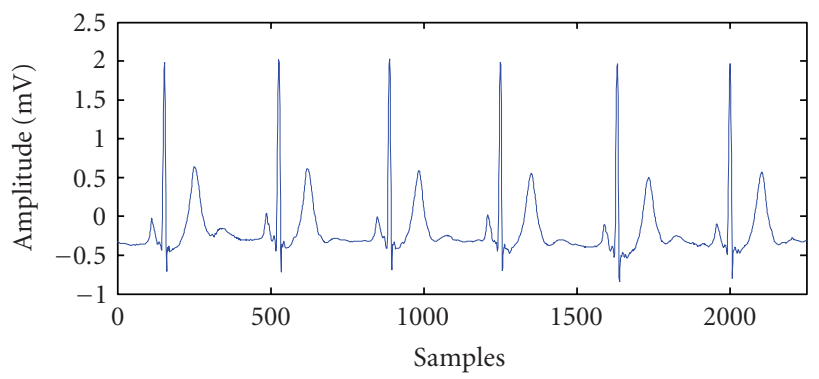

(a)

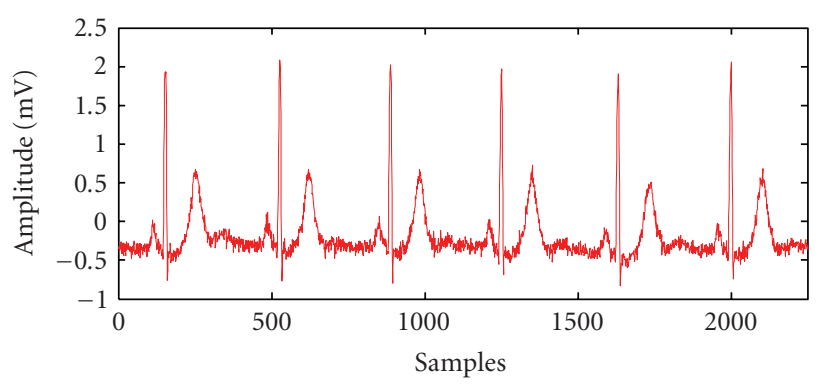

(b)

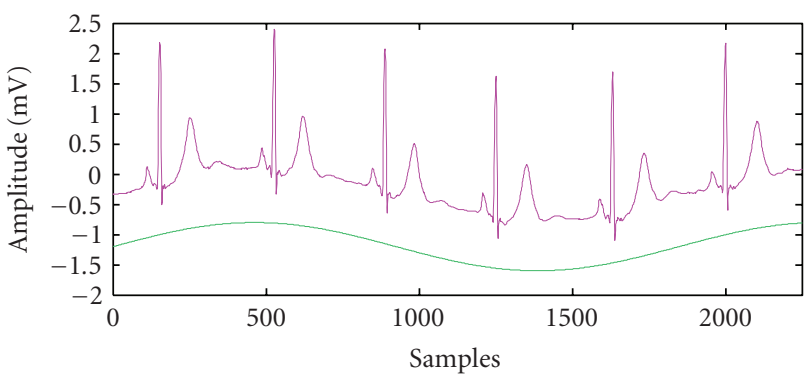

(c)

FIGURe 2: (a) Normal ECG, (b) noisy ECG, (c) baseline wandered ECG with its baseline trace.

\section{DEFINITION OF BIONIC WAVELET TRANSFORM}

The wavelet transform comprises the coefficients of the expansion of the original signal $x(t)$ with respect to a basis $h_{a, \tau}(t)$, each element of which is a dilated and translated version of a function $h(t)$, called the mother wavelet, according to

$$
h_{\tau, a}(t)=\frac{1}{\sqrt{|a|}} h\left(\frac{t-\tau}{a}\right) .
$$

Depending on the choice of the mother wavelet appropriately, the basis can be orthogonal or biorthogonal. The wavelet transform coefficients, given by the inner product of $x(t)$ and the basis functions,

$\mathrm{WT}_{x}(\tau, a)=<x(t), \quad h_{\tau, a}(t)>=\frac{1}{\sqrt{|a|}} \int x(t) h^{*}\left(\frac{t-\tau}{a}\right) d t$

comprise the time-frequency representation of the original signal. The wavelet transform has good localization in 


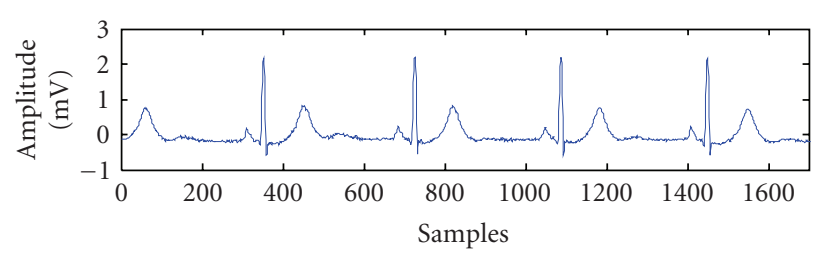

(a)

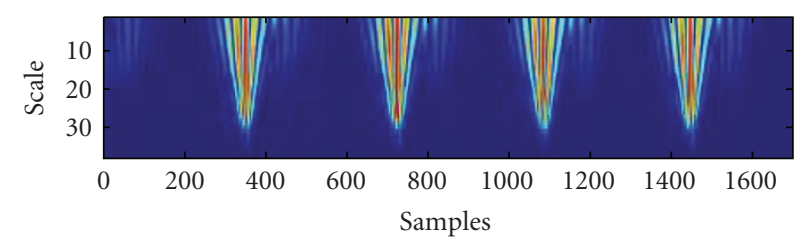

(b)

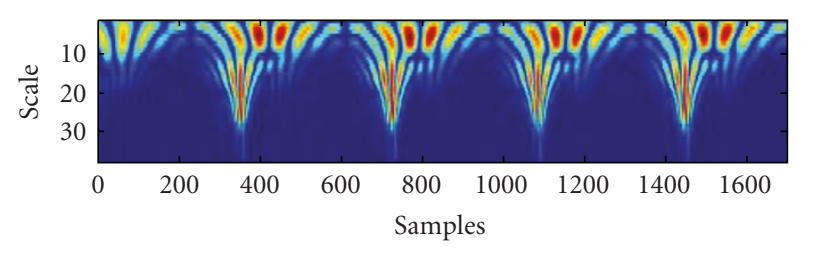

(c)

FIgURE 3: (a) MIT-BIH ECG record number 106, (b) timefrequency representation with $\mathrm{WT}$, (c) the same representation with BWT.

both frequency and time domains, having fine frequency resolution and coarse time resolution at lower frequency, and coarse frequency resolution and fine time resolution at higher frequency. Since this matches the characteristic of most signals, it makes the wavelet transform suitable for time-frequency analysis.

The concept of multiresolution analysis can be extended from the fixed wavelet mother function to the varying in the time spread case. This new approach is known as the adaptive bionic wavelet transform and would be addressed next.

The idea behind the BWT is to replace the constant quality factor of the wavelet transform with a variable adaptive quality factor [17]. To do this, one can make changes in the mother function of the wavelet transform. Satisfying the admissible condition for the mother wavelet, the oscillating $h(t)$ can be represented as

$$
h(t)=\tilde{h}(t) \exp \left(j 2 \pi f_{0} t\right)
$$

where $f_{0}$ is the center frequency of $h(t)$ and $\tilde{h}(t)$ is its envelope function. Using a $T$-value, the BWT mother function would be stated as follows:

$$
h_{T}(t)=\frac{1}{T} \tilde{h}\left(\frac{t}{T}\right) \exp \left(j 2 \pi f_{0} t\right)
$$

The BWT is now defined by the following equation [17]:

$$
\begin{aligned}
\operatorname{BWT}_{x}(\tau, a)= & \frac{1}{\sqrt{|a|}} \int x(t) h_{T}^{*}\left(\frac{t-\tau}{a}\right) d t \\
= & \frac{1}{T \sqrt{|a|}} \int x(t) \tilde{h}^{*}\left(\frac{t-\tau}{a T}\right) \\
& \times \exp \left(-j 2 \pi f_{0}\left(\frac{t-\tau}{a}\right)\right) d t
\end{aligned}
$$

As can be seen, in contrast to the wavelet transform, both the amplitude and the time spread of BWT mother function depend on the $T$ value. For evaluating the $T$ parameter, Yao and Zhang adopted a general nonlinear form based on a previously introduced auditory model. This results in the following formula for a function, namely, the $T$-function:

$$
\begin{aligned}
T(\tau+\Delta \tau)= & \left(1-\widetilde{G}_{1} \frac{\mathrm{BWT}_{s}}{\mathrm{BWT}_{s}+\left|\mathrm{BWT}_{x}(\tau, a)\right|}\right)^{-1} \\
& \times\left(1+\widetilde{G}_{2}\left|\partial \mathrm{BWT}_{x}(\tau, a) / \partial t\right|\right)^{-1}
\end{aligned}
$$

where $\widetilde{G}_{1}, \widetilde{G}_{2}$, and $\mathrm{BWT}_{s}$ are constants, and $\operatorname{BWT}_{x}(\tau, a ; h)$ is the BWT coefficient at time $\tau$ and scale $a$, and $\Delta \tau$ is the calculation step. Clearly, it is the $T$-function that brings adaptation to the BWT. For detailed information about how the $T$-function is derived and the underlying mechanism, the reader is referred to $[18,19]$.

\section{BIONIC WAVELET TRANSFORM OPTIMIZATION FOR ECG ANALYSIS}

According to the definition of BWT, there is a major difference in resolution of time-frequency span of analyzing windows. In fact, in the WT, for a fixed mother function, all the windows in a certain scale along the $t$-axis are fixed and the window size of the WT varies with the change of analyzing frequency. However, both the time and frequency resolutions can be different in the BWT even in a certain scale. The adjustment of the BWT resolution in the same scale is controlled by $T$-function, which is related to the signal instantaneous amplitude and its first-order differential [17].

Figure 3 shows the time-frequency representation for an ECG signal with both WT and BWT. Notice the smoothing in the BWT representation which is the direct result of windows changes over certain scales.

It only remains to set the BWT parameters efficiently so that it can decompose the signal into finite number of scales and afterwards, determine the most energetic ones, and choose a global or local threshold. In order to optimize the BWT parameters we have used a semioptimal method considering both analytic and morphological aspects of the analyzed signal. As we are considering ECG signal, we should be aware of its variability.

Presumably the most important feature for an ECG signal is the frequency range in which its main components occur. Although there are some other components like ventricular late potentials (VLPs), we have restricted our interest on $\mathrm{P}, \mathrm{Q}, \mathrm{R}, \mathrm{S}$, and $\mathrm{T}$. The resulting frequency range is up to $100 \mathrm{~Hz}$. 
Let $f_{0}$ be the initial center frequency of the mother wavelet. In [17] it has a value equal to $15165.4 \mathrm{~Hz}$ and as the scale goes higher and higher, the center frequency will decrease in the following way:

$$
f_{m}=\frac{f_{0}}{q^{m}}, \quad q>1
$$

For ECG we do not need such high $f_{0}$, so we optimized it simply by running the program for different values of $f_{0}$ and then minimizing the gradient of error variance by comparing the results-numerically and morphologically-with each other. It has been found that if the center frequency lies in the range of 360 to $500 \mathrm{~Hz}$ there would be no much distortion on the analyzed ECG. The reason behind this choice lies in the fact that to have no aliasing it is preferred to choose the center frequency of the first scale a value more than ECG sampling frequency. Here we have chosen $f_{0}=400$ which yields satisfactory results.

Unlike [17], in our method $q$ is not a global constant, but for each signal and scale of decomposition it takes a fixed value which should obey an adaptation procedure. Besides for every $m$, that is, in each distinct scale, it is adapted for different time-frequency windows. More explanation on how $q$ is determined according to every analyzing window is to be given in the next section. Other parameters are exactly the same as what was stated in $[20,21]$ which used BWT for speech enhancement and denoising. These constants are $\widetilde{G}_{1}=0.87, \widetilde{G}_{2}=45$, and $\mathrm{BWT}_{s}=0.8$.

Finally, the calculation step is determined due to the sampling frequency. If we let $f_{s}$ be the sampling frequency, then the step will be $\Delta \tau=1 / f_{s}$.

\section{APPLICATION TO DENOISING AND BASELINE WANDERING REDUCTION}

For investigating the applications of MABWT to ECG signal, we have restricted our interest on denoising and baseline wandering reduction. In each of the following sections, we have proposed modifications to BWT so as to be optimized for the field of analysis. For baseline wandering elimination, the goal is optimizing BWT by adopting a multiadaptation scheme in which the low passed wandering is automatically removed and there is no need for extra processing on distinct subbands.

\subsection{Denoising}

After BWT optimization, the denoising technique illustrated in Figure 4 is used to reduce the amount of noise contamination in the ECG signal. In implementation, BWT coefficients can be easily calculated based on corresponding WT coefficients by

$$
\operatorname{BWT}_{x}(\tau, a ; T)=K \times \mathrm{WT}_{x}(\tau, a),
$$

where $K$ is a factor depending on $T$ [18]. Especially, for the real Morlet function $h(t)=e^{-\left(t / T_{0}\right)^{2}}$, which is used as the

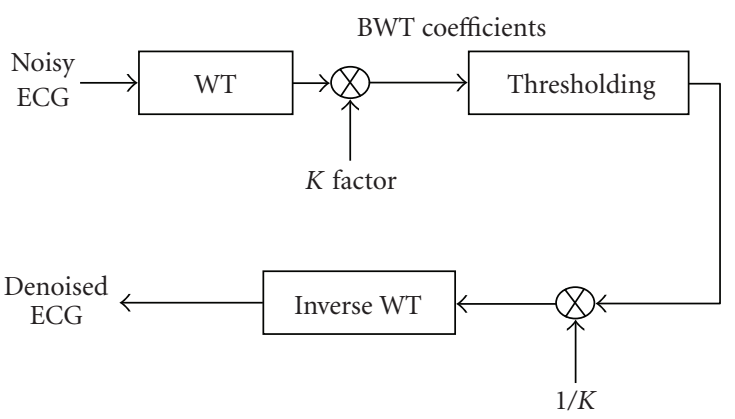

FIGURE 4: Block diagram of the bionic wavelet transform denoising technique.

mother function in our experiments, $K$ is equal to [21]:

$$
\frac{\int_{-\infty}^{+\infty} e^{-t^{2}} d t}{\sqrt{\left(T / T_{0}\right)^{2}+1}} \approx \frac{1.7725}{\sqrt{\left(T / T_{0}\right)^{2}+1}}
$$

Here we have used Donoho's proposed approach for denoising including two major categories, hard thresholding and soft thresholding $[10,22]$. Choosing the threshold value can be determined in many ways. Donoho derived the following formula based on white Gaussian noise assumption:

$$
\text { thr }=\sigma \sqrt{2 \log _{2} N},
$$

where thr is the threshold value, $N$ is the length of the noisy signal, and $\sigma=$ AMFS $/ 0.6745$, with AMFS denoting the absolute median estimated on the first scale of the bionic wavelet coefficients.

Knowing the fact that it is the $T$-function (or the $K$ factor, equivalently) which results in the adaptive characteristic of the BWT, it may be a good idea to use the values of the $T$-function to come to a new thresholding rule. On the other hand, it is expected for the BWT coefficients to take small values in the higher scales of decomposition with regard to (8), the same as the WT coefficients. Thus it seems to be logic to use the values of the $T$-function in lower scales of decomposition. Combining the above idea with Donoho's formula yields the following threshold which is a new modification of (10). Let $T_{\mathrm{fs}}(i)$ be the value of $T$-function in the first step of computing. The threshold is formulated as

$$
\text { thr }=\frac{\sigma}{\sum_{i} \alpha_{i} T_{\mathrm{fs}}(i)} \sqrt{2 \log _{2} N} .
$$

In fact a weighted average of $T$-function values in the first scale of decomposition with the bionic wavelet transform is added to (10) to get better results. The $\alpha_{i}$-weights are chosen with try and error for the algorithm to have the highest performance. But an experimentally right choice is to let the $\alpha_{i}$ coefficients be a decreasing function.

Yao showed that if the signal and its first-order differential are continuous, BWT can reconstruct the original signal without distortion [17]. In the current approach and after thresholding, the coefficients of BWT are divided by $K$-factor followed by an inverse WT transform which reconstructs the denoised version of the signal. 


\subsection{Baseline wandering reduction}

Among various methods used for baseline wandering reduction, an efficient technique was proposed by Park et al. [23]. They presented a wavelet adaptive filter (WAF) which consists of two parts, in the first part the signal is decomposed into seven subbands and in the second part the seventh lowest band subsignal is adaptively filtered. But the method suffers from preserving the signal quality, specially in the S-T segment.

Another approach which has utilized the wavelet packets to eliminate the ECG baseline wandering was introduced, which removes the components that are not correlated to ECG and have such characteristics that are somehow added to it [16]. But the method does not fully take the advantage of interbeat correlation of the ECG signal. Hence, baseline drifts that occur occasionally and cannot be considered to be an added source to the ECG would not be removed efficiently.

As for the case of BWT, the resolution in the timefrequency domain can be adaptively adjusted not only by the signal frequency but also by the signal instantaneous amplitude and its first-order differential. Hence, analyzing the ECG signal in the time-frequency plane of BWT not only has a good chance to remove the baseline wanderings but also has promising results preserving the clinical information of the ECG record.

Since the spectrum of the baseline is below the spectrum of the ECG signal, therefore its energy concentration in corresponding time-scale plane does not change much as the scale is changed in the binary decomposition tree, but the energy of the ECG signal decreases as the scale is changed. Therefore, in the binary tree search we reach a point that the energy of the ECG signal almost vanishes (no details in that scale) but we still have considerable energy for baseline wandering.

Using the multiadaptive bionic wavelet transform makes possible the detection and reduction of ECG baseline wandering in low frequency subsignals. As we mentioned before the central frequency of each scale is adaptively chosen. For adaptation purpose, we have used the following criteria based on the time-frequency representation of the analyzed signal under the adaptive BWT.

If we suppose that the baseline wandering can be estimated by a sinusoidal function (see Figure 2(c)), the frequency of the sine function can be approximately estimated using the spectrum of the signal, in which we should seek for a peak. In most cases the second distinguished peak in the Fourier transform of the signal corresponds to the wandering frequency. For ECG signals with the baseline wanderings which cannot be considered as sinusoidal, the $f_{w}$ estimation is obtained from the time-frequency representation of the signal. In this case a frequency exists all over the time which deals with the baseline trace. After determining an estimation of the baseline wandering frequency, $f_{w}$, we may use the thresholding rule of (11) for the three consecutive scales, the one that contains $f_{w}$, the previous and the next scales. To evaluate the coefficients of the multiadaptive bionic wavelet transform we need the value of $q$. To determine the value of $q$ we remember the fact that the mean of the baseline wandered ECG and the mean of the baseline corrected ECG have the maximum distance. Thus we use the maximum distance (MD) criteria to verify the optimal $q$ for which the distance between the MABWT coefficients of original signal and the baseline corrected signal is maximized. We define the MD criteria as

$$
\begin{aligned}
q_{\mathrm{opt}}=\arg \max _{q}\{ & \left(\mid \operatorname{MABWT}_{x}(\tau, a)\right. \\
& \left.\left.-\left.\operatorname{MABWT}_{\hat{x}}(\tau, a)\right|^{2}\right)_{(\tau, a) \in \mathfrak{I}^{m}}\right\},
\end{aligned}
$$

where $x$ and $\hat{x}$ are the original and processed signals, $m$ is the scale number $\left(a=2^{-m}\right)$, and $\mathfrak{I}^{m}$ denotes the analyzing window of the $m$ th scale which is centered at $f_{w}$. Solving the above optimization problem, we will have $q$ for the next scale. Although $q$ is optimally selected, the number of scales $m$ remains to be determined according to the $q$ value of the previous scale. In other words, we begin with $m=1$. For the first scale, that is, $m=1$, MABWT is computed using an initial value for $q$. Then (12) gives the optimum value of $q$ for the next scale, $m=2$. The procedure proceeds iteratively until the center frequency of the analyzing window, which is defined according to both $m$ and $q$ (refer to (7)), goes beyond the desired predefined ECG frequency range.

The step by step multiadaptation together with the adapted $T$-function (or $K$-factor) of BWT can cope with the problem of ECG baseline wandering reduction better than WT. Furthermore as we have an estimation of $f_{w}$, the adaptation can be used only in three successive scales in which the mid-scale has the closest center frequency to $f_{w}$. So the implementation is possibly time consuming.

\section{SIMULATION RESULTS}

To show that MABWT is appropriate for ECG denoising we have used two types of ECG signals, both simulated and real ones. We used the MIT-BIH arrhythmia database [24] as the reference for our real signals, all with sampling frequencies, $f_{s}$, equal to $360 \mathrm{~Hz}$. For the simulated ECGs we have used the dynamical model which was introduced for generation of synthetic ECG cardiac signals [25, 26].

The signals were decomposed using MABWT up to 40 scales depending on the different values of $q$ that was optimally selected using (12). We have used Morlet wavelet as mother wavelet and its support length is chosen as $[-4,4]$, and $2.5 \pi$ is chosen as its oscillatory frequency [21]. For simulation, we have chosen $f_{0}=400 \mathrm{~Hz}\left(f_{0}>f_{s}\right)$. In addition we have considered the simplest case for our new thresholding rule, (11). Hence, we have set $\alpha_{i}=1 / i$.

\subsection{Denoising}

In order to investigate the performance of various methods, artificial white Gaussian noises with different variances were generated and added to the test signals. As mentioned before two kinds of thresholding methods, hard and soft, were applied for denoising based on the modified thresholding rule introduced by (11). Besides, for easier comparison we have 
applied the wavelet-based denoising with Daubechies (db4) wavelets to each signal and we have shown the results for both hard and soft thresholdings. Figures 5-7 show some typical results, considering real and simulated ECG signals of two general categories, that is, normal and abnormal ones. One can see that in many cases, hard thresholded signal is much similar to the soft thresholded ECG, which is due to the intrinsic smoothness in BWT.

Simulation results provide supportive evidence to claim that MABWT has some advantages over the traditional WT for ECG denoising. First, it has higher sensitivity so it is more probable for WT to have little single noise samples (speckles) remained. Second, MABWT has a smoothing property with respect to its resolution variation over the time-frequency plane, and this is exactly what we are seeking in many denoising techniques. This is particularly true for real ECGs (see Figure 6). Furthermore, the effect of the adaptation is clearly obvious in the first samples of the reconstructed ECGs with MABWT (refer to Figures 5(e) and 5(f)). But as the adaptation proceeds, the reconstructed signal follows the clean ECG morphology.

Another field of interest for the proposed multiadaptive method is to denoise and reconstruct abnormal signals. Figure 7(a) shows an ectopic beat among a normal ECG cycle. We have applied the MABWT to the signal and have shown the results corresponding to hard MABWT (HBWT). It is clear that the denoised signal is noise-free but cannot follow the ST segment of the ectopic beats, reasonably because any abnormality corresponds to an abrupt change in the signal rhythm and consequently the adaptation needs time to follow this behavior (Figure 7(b)). But fortunately this does not affect the diagnostic features of the ECG signal such as the QT or the ST intervals, and the QRS complex.

Since we have used the adaptation in a limited number of scales, abrupt changes may be tracked not efficiently, but it can be shown that if the adaptation includes all decomposition subbands and also the abnormality can be considered semicyclic, but not necessarily stationary, the MABWT technique would come over the above problem (refer to Figure 7(c)).

For evaluating the performance of the proposed BWT we have used the SNR improvement measure by the means of the expression:

$$
\begin{aligned}
\operatorname{imp}[\mathrm{dB}] & =\mathrm{SNR}_{\text {output }}-\mathrm{SNR}_{\text {input }} \\
& =10 \log \left(\frac{\sum_{i}\left|x_{d}(i)-x(i)\right|^{2}}{\sum_{i}\left|x_{n}(i)-x(i)\right|^{2}}\right),
\end{aligned}
$$

where $x$ denotes the original ECG, $x_{d}$ is the denoised signal, and $x_{n}$ represents the noisy ECG signal. Figure 8 compares the improvement values between WT and MABWT. For evaluation, we have chosen first 4096 samples of the noise free MIT-BIH record number 100 as our reference ECG signal. As mentioned before, both hard and soft thresholdings have been considered for denoising. One can see that in lower input SNRs, soft MABWT (SBWT) has a better performance and as the input SNR is increased SBWT remains the best choice with improvements much more than that of WT.

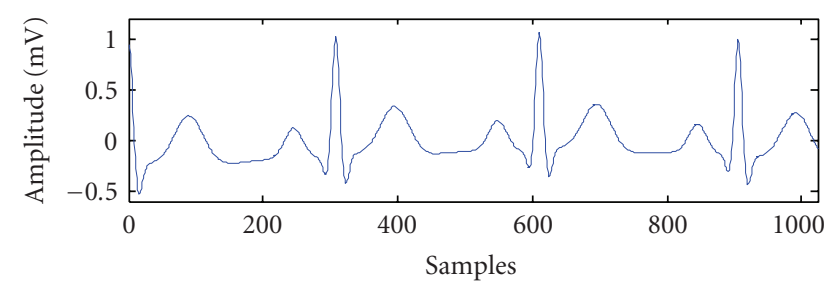

(a)

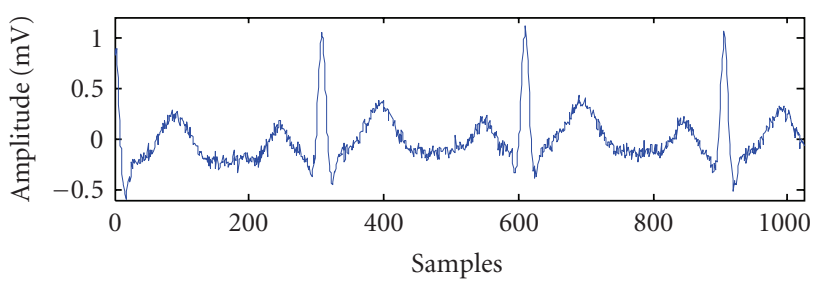

(b)

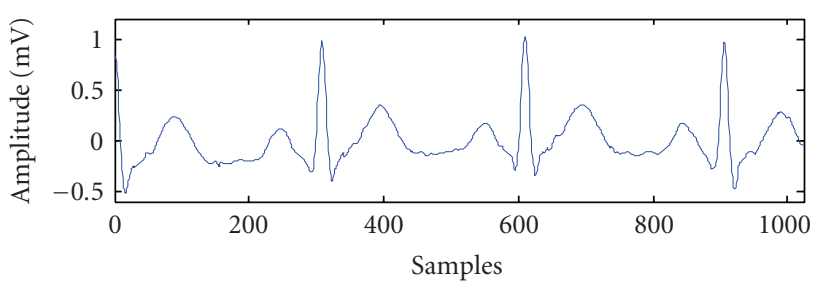

(c)

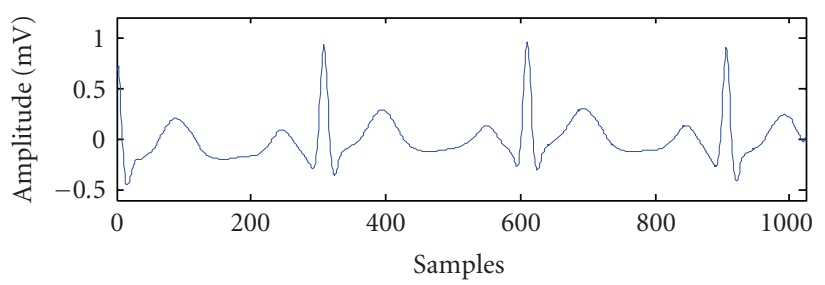

(d)

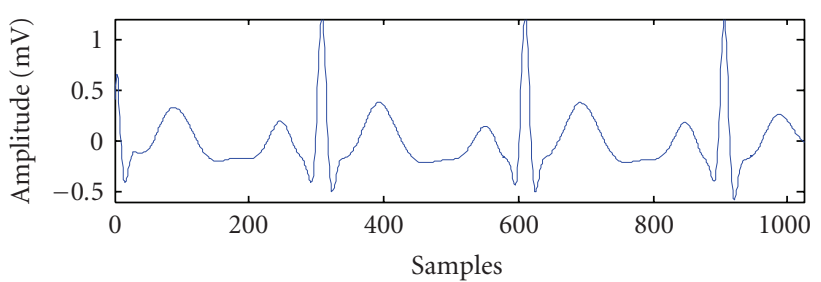

(e)

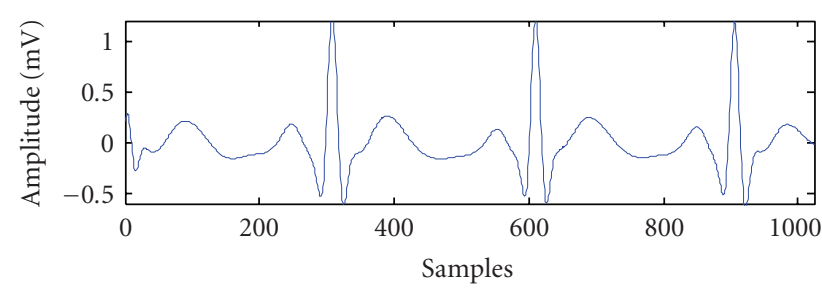

(f)

FIgUre 5: Typical results of different methods for an input simulated signal of $6 \mathrm{~dB}$. (a) Clean ECG, (b) noisy input signal, (c) WT (hard), (d) WT (soft), (e) MABWT (hard), and (f) MABWT (soft). 


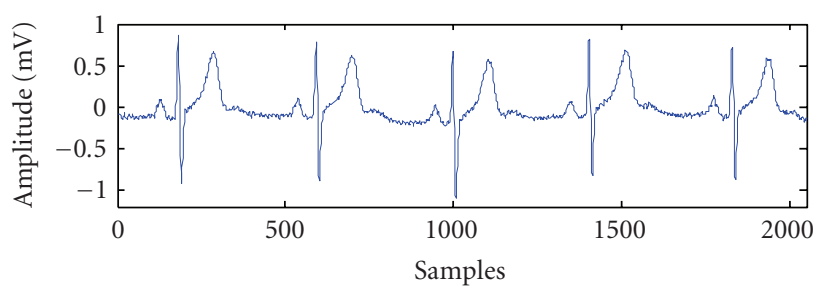

(a)

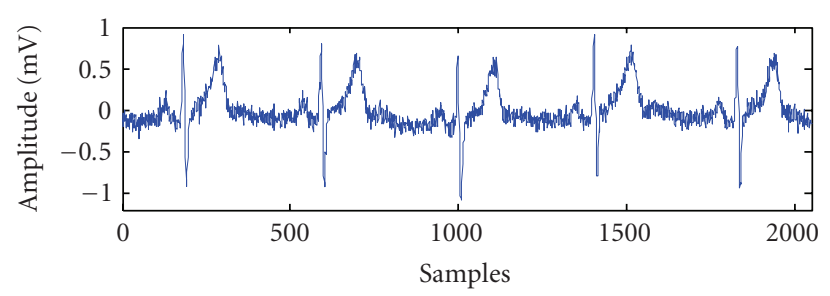

(b)

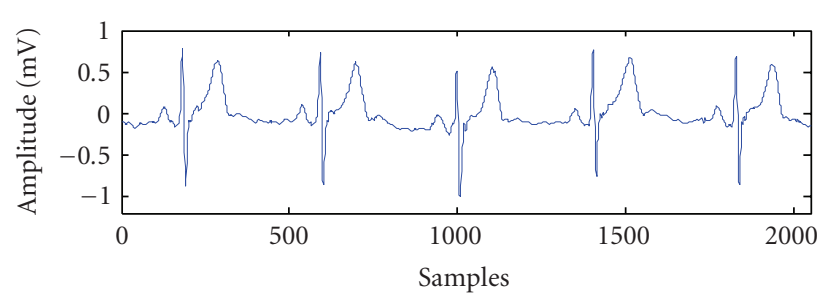

(c)

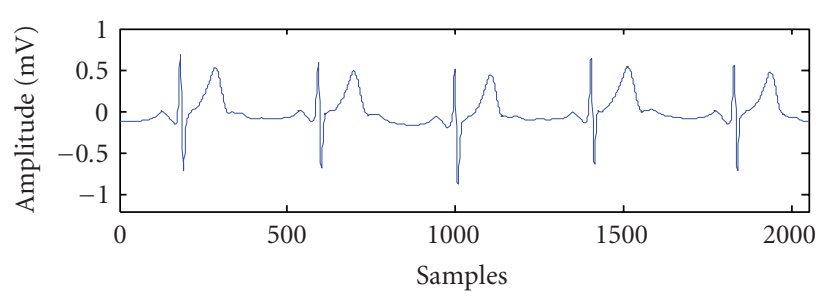

(d)

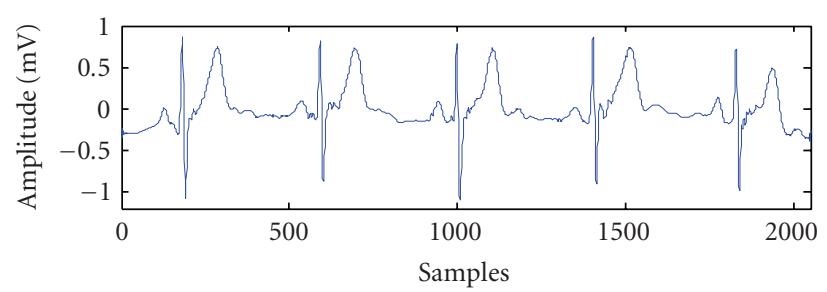

(e)

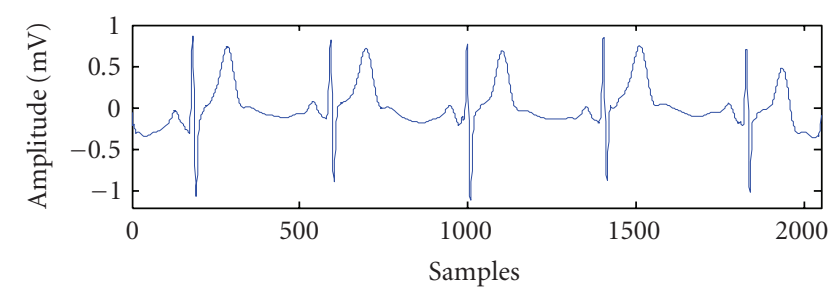

(f)

FIgURE 6: Typical results of different methods for the MIT-BIH record 117 with an input SNR of $4 \mathrm{~dB}$. (a) Clean ECG, (b) noisy input signal, (c) WT (hard), (d) WT (soft), (e) MABWT (hard), and (f) MABWT (soft).

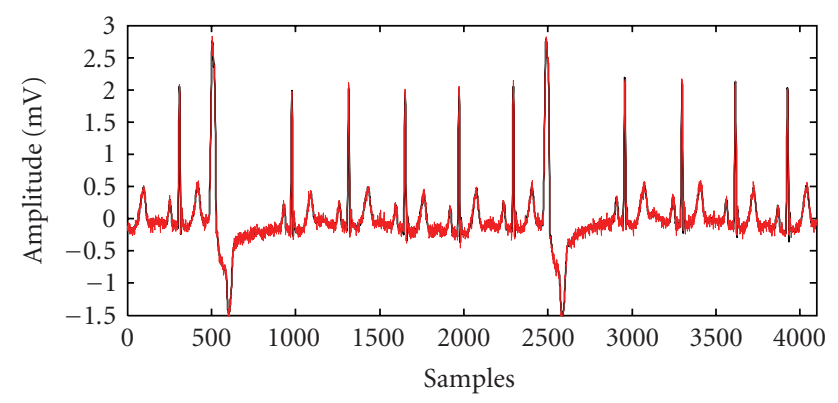

(a)

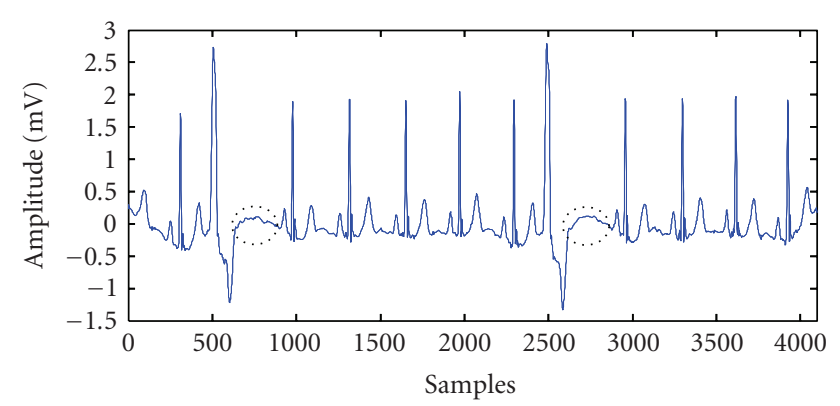

(b)

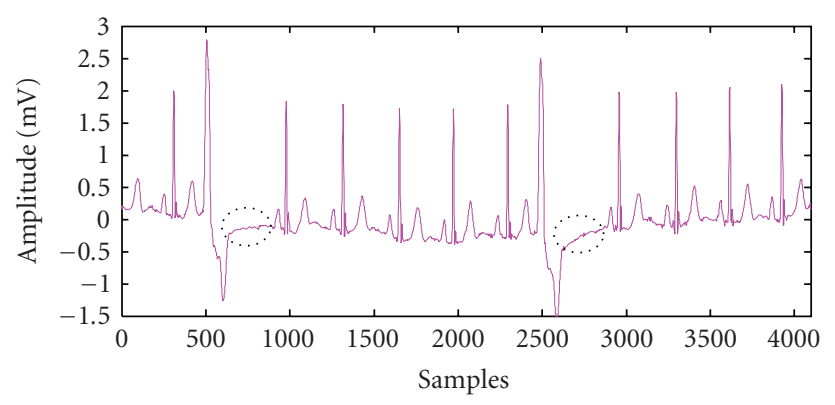

(c)

FIGURE 7: Denoising results of HBWT applied to an ECG (MIT-BIH record 119) with ectopic beats. (a) Clean and noisy ECG, (b) HBWT with 3 levels of adaptation, (c) HBWT with the adaptation applied to all decomposition levels. The surrounding circles indicate the ST segment of the ectopic beats.

Moreover, HBWT acts better than hard WT and for SNRs higher than $8 \mathrm{~dB}$, it passes the soft WT performance. Figure 8 clarifies that for a wide range of input SNRs, the MABWT improvement (hard or/and soft) has a noticeable difference to that of WT, especially outstanding for lower SNRs for which having nearly $2 \mathrm{~dB}$ improvement in MABWT is of major importance.

In order to have a comparison between the performance of the proposed method and conventional wavelet-based ECG denoising schemes, WT and the MABWT were tested on the database. The results of the SNR improvement for the input SNR of $5 \mathrm{~dB}$ are listed in Table 1.

According to these results the MABWT performance is always better than the corresponding WT for hard and soft thresholdings, as was already expected. Although WT and 


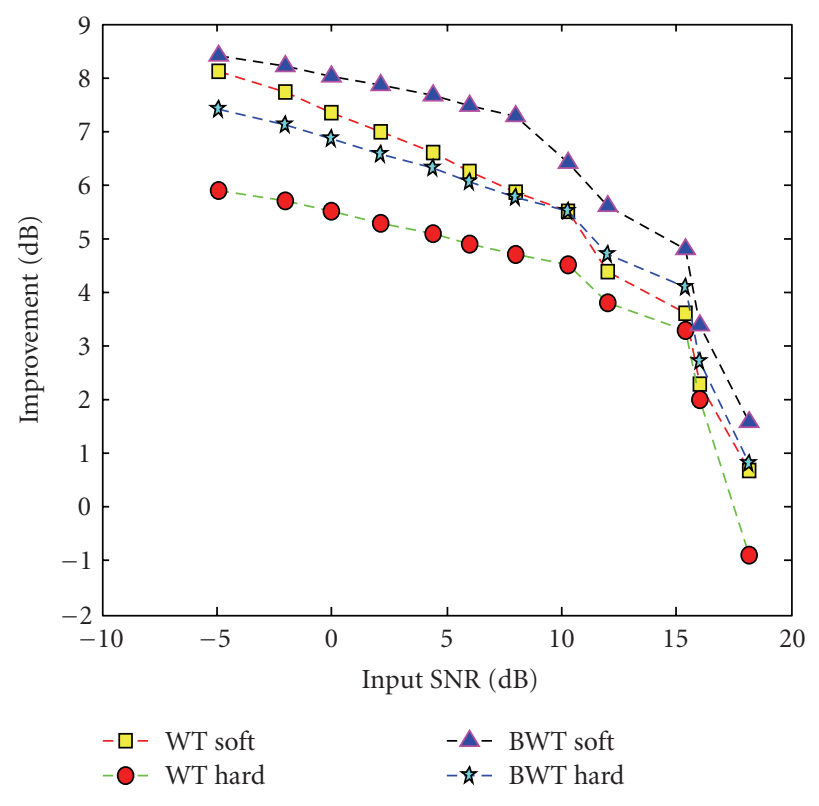

FIGURE 8: WT and MABWT filter output SNR improvement versus different input SNRs for the first 4096 samples of the MIT-BIH record number 100 .

MABWT always improve the input SNR, MABWT with soft thresholding has the best overall performance with a maximum SNR improvement of $8.2 \mathrm{~dB}$, while the best result of WT-based approach goes back to $6.6 \mathrm{~dB}$.

\subsection{Baseline wandering reduction}

We have divided the baseline wanderings into the following two general categories: DC components affecting the baseline not to be at zero level, and baseline drift which is a consequence of low frequency interferences. For both cases with the appropriate choices for the level of decomposition, that is, number of scales $m$, and the center frequency $f_{0}$, removing baseline wandering would be a direct task. Remember that we have assigned a fixed value to $f_{0}$, experimentally. On the other hand there is an interdependency between $m$ and $q$ (7) but with the help of the iterative algorithm discussed in Section 4, there would be only one degree of freedom to determine the accurate values of the mentioned parameters. Again it only remains to find $q$ with respect to the optimization problem of (12) for $m=2$ and continue with the computed value(s) for the next scale(s). To clarify the effectiveness of the proposed technique, we have chosen real ECG signals including two classes of baseline wandering simultaneously, and we have run the baseline wandering cancellation algorithm with both WT and MABWT. The results are provided in Figures 9-11.

Results show that although for the case of wavelet transform the denoised signal is somehow free of the baseline interference, but the drifts still exist unless applying a lowpass filtration to the properly selected subband (Figure 9(b)). Moreover, the thresholding not only affects the baseline but
TABLE 1: WT and MABWT denoising performance on the MIT-BIH arrythmia database.

\begin{tabular}{|c|c|c|c|c|}
\hline \multirow{2}{*}{$\begin{array}{l}\text { Record } \\
\text { no. }\end{array}$} & \multicolumn{4}{|c|}{ SNR improvement (dB) } \\
\hline & $\begin{array}{c}\text { WT } \\
\text { (hard) }\end{array}$ & $\begin{array}{c}\text { WT } \\
\text { (soft) }\end{array}$ & $\begin{array}{c}\text { MABWT } \\
\text { (hard) }\end{array}$ & $\begin{array}{c}\text { MABWT } \\
\text { (soft) }\end{array}$ \\
\hline 100 & 5.1 & 6.5 & 6.4 & 7.8 \\
\hline 101 & 4.2 & 5.5 & 5.3 & 6.9 \\
\hline 103 & 5.0 & 6.1 & 5.8 & 7.7 \\
\hline 105 & 5.1 & 6.0 & 5.8 & 8.1 \\
\hline 112 & 5.2 & 6.4 & 6.1 & 8.2 \\
\hline 113 & 5.0 & 6.2 & 5.9 & 7.9 \\
\hline 115 & 5.1 & 6.6 & 6.3 & 7.8 \\
\hline 116 & 5.0 & 6.5 & 6.4 & 8.0 \\
\hline 117 & 4.8 & 6.0 & 5.8 & 7.9 \\
\hline 119 & 4.7 & 5.8 & 5.6 & 7.6 \\
\hline 122 & 4.4 & 5.6 & 5.2 & 6.9 \\
\hline 123 & 5.1 & 6.5 & 6.4 & 7.8 \\
\hline 200 & 4.2 & 5.4 & 5.3 & 6.9 \\
\hline 201 & 5.0 & 5.4 & 5.5 & 7.5 \\
\hline 202 & 4.9 & 5.8 & 5.7 & 7.8 \\
\hline 205 & 5.0 & 5.4 & 5.5 & 7.4 \\
\hline 209 & 5.2 & 6.0 & 5.9 & 8.1 \\
\hline 210 & 4.3 & 5.4 & 5.3 & 6.9 \\
\hline 212 & 4.0 & 5.0 & 4.8 & 6.7 \\
\hline 213 & 5.2 & 6.6 & 6.4 & 8.2 \\
\hline 219 & 5.2 & 6.5 & 6.3 & 8.0 \\
\hline 220 & 5.1 & 6.5 & 6.4 & 8.1 \\
\hline 221 & 4.9 & 6.0 & 5.9 & 7.9 \\
\hline 230 & 5.0 & 5.9 & 5.6 & 7.9 \\
\hline 233 & 4.8 & 5.8 & 5.5 & 7.8 \\
\hline Average & 4.86 & 5.98 & 5.80 & 7.67 \\
\hline
\end{tabular}

also has destructive effects on the signal morphology, especially on the ST segment, which is extremely sensitive to noise and is of great clinical significance (Figure 10(b)). But in the denoised signal with MABWT, baseline wandering is completely removed and also a noise-free version of the signal is obtained without the need for any extra filtering. There are more with the advantages of MABWT over other baseline correction techniques. It is capable of eliminating not only those drifts which could be modeled as additive sources [16], but also the ones that have no correlation with a pure ECG. In addition, the method has no destructive effects on the morphology of the signal and can cope with both normal and abnormal beats, while previously introduced methods have problems with these cases [8].

The results are also presented for simulated baseline perturbations, as depicted in Figure 12. We have chosen the MIT-BIH record number 207, which includes bundle branch blocks, together with ventricular flutter wave. A random noise of $6.5 \mathrm{~dB}$ was added to evaluate the noise reduction performance of the algorithm, while reducing the baseline drift. The difference between processed and original signal is shown for soft thresholding. It shows that WT processed 


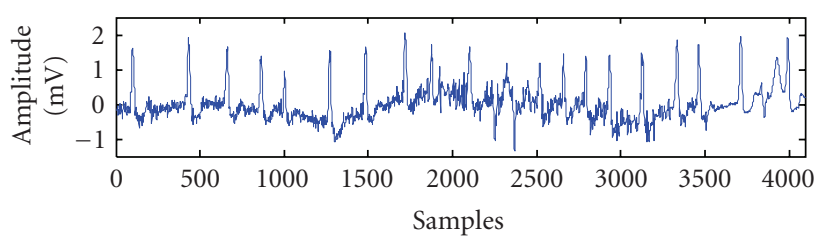

(a)

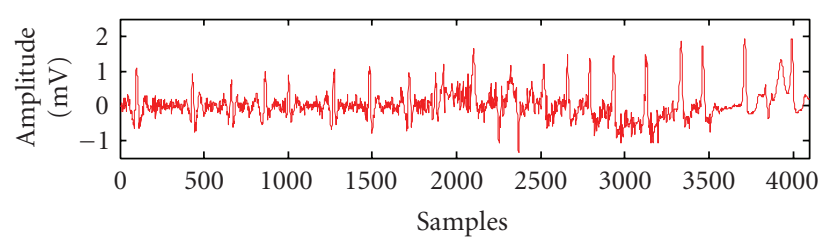

(b)

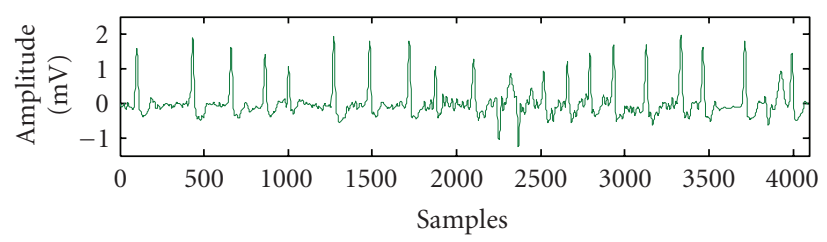

(c)

FIgURE 9: Typical results of different methods for baseline correction in presence of DC components and lowpass interference. (a) MIT-BIH record 203, (b) result of applying WT, (c) result of applying MABWT.

signal has its maximum difference on the QRS complexes and the T waves. In contrast to WT, the processed signal with BWT, when adapted to the signal, follows its morphology, leading the difference to be ignored. Consequently, WT cannot handle precise baseline wander removal, and there is a significant difference in the amplitude of the fiducial points of the signal, compared to the original ECG. Hence, there may be distortions in the locations of the ECG points and interval features.

\section{DISCUSSION AND CONCLUSION}

We have presented and validated a new multiadaptive version of the bionic wavelet transform and its applications to noise and baseline wandering suppression in electrocardiograms combined with modifications of the traditional thresholding rules. The MABWT aims at integrating into the standard BWT a mechanism that adjusts the center frequency of every analyzing scale in a signal-adaptive fashion. Moreover, the value of the $T$-function, which controls the mother wavelet, is directly influenced on the threshold to come to an appropriate criterion for the denoising approach which uses the transform coefficients' information.

To show that BWT improvement is really effective in clinical situations, the method has been validated using several ECG recordings with a wide variety of wave morphologies from MIT-BIH arrhythmia database, and also simulated signals. For denoising purpose, using the MABWT representa-

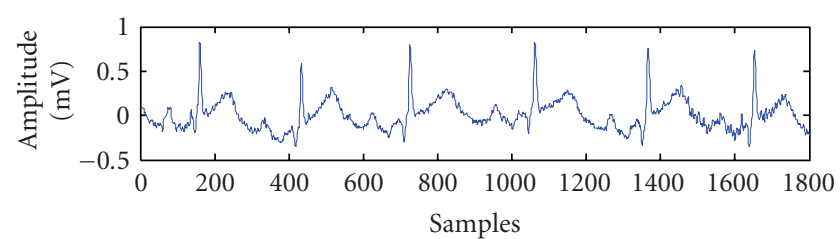

(a)

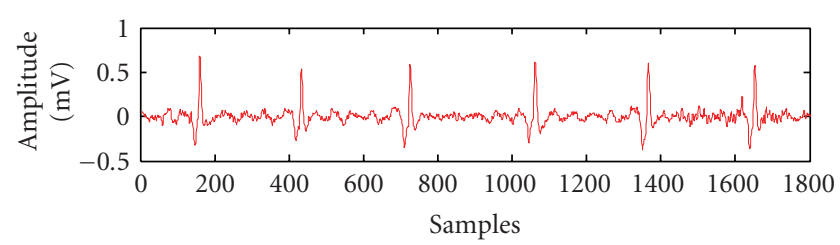

(b)

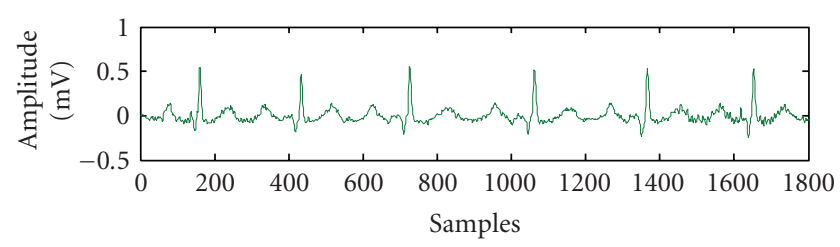

(c)

FIGURE 10: Typical results of different methods for baseline wandering cancellation in presence of sinusoidal drifts. (a) MIT-BIH record 228, (b) result of applying WT, (c) result of applying MABWT.

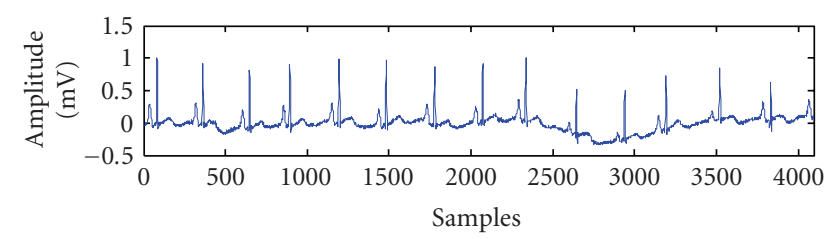

(a)

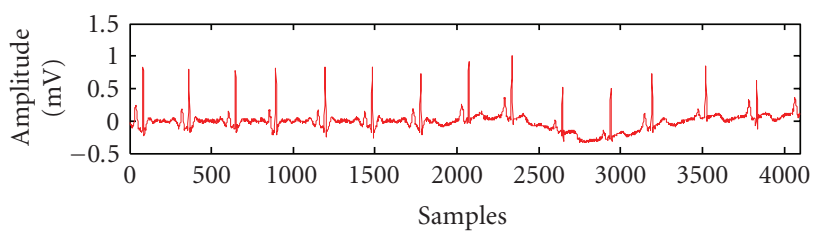

(b)

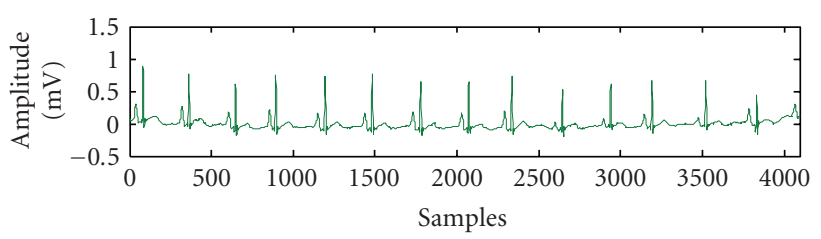

(c)

Figure 11: Typical results of different methods for baseline wandering elimination for a non-correlated baseline artifact. (a) MIT$\mathrm{BIH}$ record 222, (b) result of applying WT, (c) result of applying MABWT. 


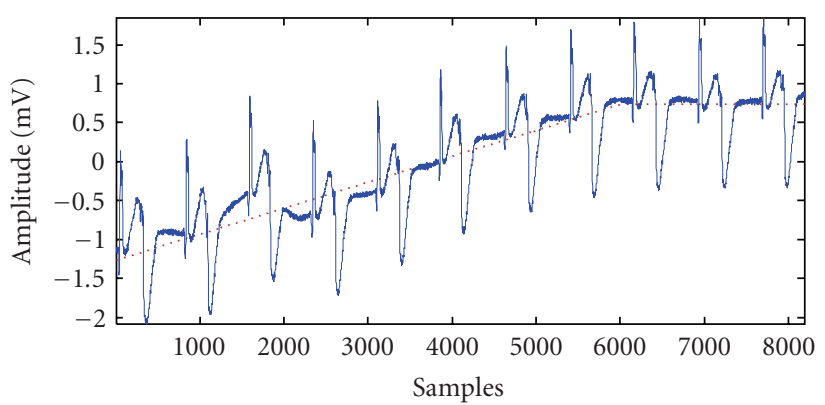

(a)

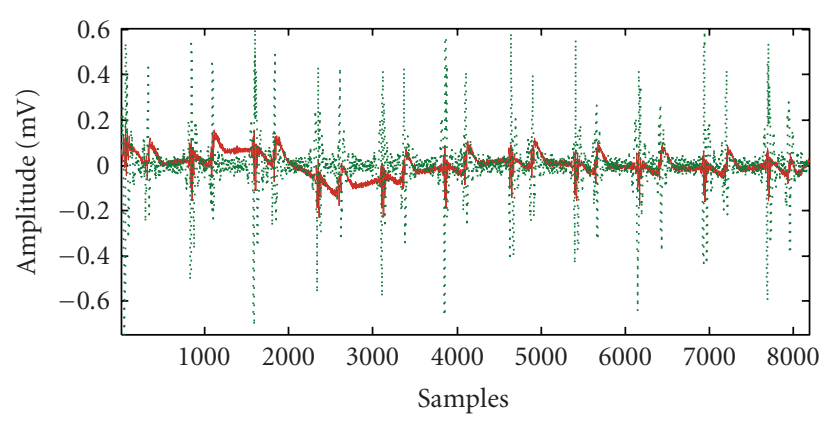

(b)

FIGURE 12: Simulated baseline perturbation removal and noise reduction for an abnormal ECG. (a) MIT-BIH record 207, and the added baseline trace, (b) differences between WT (dashed line) and MABWT (solid line) processed signals and the original ECG.

tion of the ECG signal and with the help of the modified threshold (11) there was an average SNR improvement of $5.80 \mathrm{~dB}$ for hard thresholding and $7.67 \mathrm{~dB}$ for soft thresholding, corresponding to $0.94 \mathrm{~dB}$ and $1.69 \mathrm{~dB}$ improvement, respectively, compared to the WT-based methods, on the assumption of additive white Gaussian noise sources. None of the more complex test cases result in an improvement less than $4.8 \mathrm{~dB}$. The MABWT-based denoising, in contrast to most denosing methods found in the literature, allows taking the advantage of characteristics of adaptive time-scale analysis. Hence, there are advantages with the suggested technique compared to conventional other denoising methods; there are fewer speckles remaining in the denoised signal, and the reconstructed signal follows a smoothing behavior. In contrast to other efficient denoising techniques, abrupt changes that occur in most abnormal cases would not be tracked unless the adaptation includes all subbands. Consequently, MABWT is able to outperform other algorithms and the modified threshold selection rule, which uses the information of the transform coefficients in the first scale of decomposition, has a noise reduction ratio well within the acceptable range.

Also, the proposed MABWT algorithm removes baseline wandering, while preserving the clinical information and the morphology of the ECG record. Unlike other baseline correction schemes, it removes the components of all wandering classes that are not correlated to ECG and have such char- acteristics that are somehow added to it, DC components, and low frequency interfering drifts. Moreover, the proposed algorithm is also capable of improving signal-to-noise ratio while eliminating the baseline wandering.

The superior performance of the proposed technique is a direct result of the multiadaptive scheme, which gives the opportunity to study different types of noise through the intuitive modification of BWT parameters. Moreover MABWT can be calculated using WT by adding a $K$-factor which results in a fast implementation of the proposed algorithm.

Finally, it should be mentioned that the importance of the multiadaptive technique goes beyond the single problem of ECG denoising since it is applicable to every type of data considering a case-dependent optimization. In fact, once the time-frequency features of the data are extracted, the problem obtains an abstract representation on which it is possible to apply whichever experience matured in MABWT.

\section{ACKNOWLEDGMENTS}

The authors wish to thank Mr. Reza Sameni for his assistance and deep review of the paper. This work has been supported, financially and intellectually, by the Iran Telecommunication Research Center (ITRC), under Grant no. T/500/15023.

\section{REFERENCES}

[1] Z. Frankiewicz and E. Piêtka, "Komputerowe eliminacja linii izoelektrycznej z sygnau EKG," Problemy Techniki Medycznej, vol. 16, no. 1, 1985.

[2] N. V. Thakor and Y.-S. Zhu, "Applications of adaptive filtering to ECG analysis: noise cancellation and arrhythmia detection," IEEE Transactions on Biomedical Engineering, vol. 38, no. 8, pp. 785-794, 1991.

[3] P. Laguna, R. Jane, O. Meste, et al., "Adaptive filter for eventrelated bioelectric signals using an impulse correlated reference input: comparison with signal averaging techniques," IEEE Transactions on Biomedical Engineering, vol. 39, no. 10, pp. 1032-1044, 1992.

[4] G. B. Moody and R. G. Mark, "QRS morphology representation and noise estimation using the Karhunen-Loève transform," in Proceedings of Computers in Cardiology, pp. 269-272, Jerusalem, Israel, September 1989.

[5] A. K. Barros, A. Mansour, and N. Ohnishi, "Removing artifacts from electrocardiographic signals using independent components analysis," Neurocomputing, vol. 22, no. 1-3, pp. 173-186, 1998.

[6] T. He, G. D. Clifford, and L. Tarassenko, "Application of ICA in removing artefacts from the ECG," to appear in Neural Processing Letters.

[7] G. D. Clifford and L. Tarassenko, "One-pass training of optimal architecture auto-associative neural network for detecting ectopic beats," Electronics Letters, vol. 37, no. 18, pp. 11261127, 2001.

[8] K. Daqrouq, "ECG baseline wander reduction using discrete wavelet transform," Asian Journal of Information Technology, vol. 4, no. 11, pp. 989-995, 2005.

[9] H. A. Kestler, M. Haschka, W. Kratz, et al., "De-noising of high-resolution ECG signals by combining the discrete wavelet transform with the Wiener filter," in Proceedings of Computers in Cardiology, pp. 233-236, Cleveland, Ohio, USA, September 1998. 
[10] D. L. Donoho, "De-noising by soft-thresholding," IEEE Transactions on Information Theory, vol. 41, no. 3, pp. 613-627, 1995.

[11] M. Popescu, P. Cristea, and A. Bezerianos, "High resolution ECG filtering using adaptive BSayesian wavelet shrinkage," in Proceedings of Computers in Cardiology, pp. 401-404, Cleveland, Ohio, USA, September 1998.

[12] P. M. G. Agante Da Silva and J. P. Marques De Sá, "ECG noise filtering using wavelets with soft-thresholding methods," in Proceedings of Computers in Cardiology, pp. 535-538, Hannover, Germany, September 1999.

[13] C. R. Meyer and H. N. Keiser, "Electrocardiogram baseline noise estimation and removal using cubic splines and statespace computation techniques," Computers and Biomedical Research, vol. 10, no. 5, pp. 459-470, 1977.

[14] P. W. MacFarlane, J. Peden, J. Lennox, M. P. Watts, and T. D. Lawrie, "The Glasgow system," in Trends in ComputerProcessed Electrocardiograms: Proceedings of the IFIP Working Conference on Trends in Computer-Processed Electrocardiograms, pp. 143-150, North-Holland, New York, NY, USA, 1977.

[15] J. A. Van Alste and T. S. Schilder, "Removal of base-line wander and power-line interference from the ECG by an efficient FIR filter with a reduced number of taps," IEEE Transactions on Biomedical Engineering, vol. 32, no. 12, pp. 1052-1060, 1985.

[16] B. Mozaffary and M. A. Tinati, "ECG baseline wander elimination using wavelet packets," Transactions on Engineering, Computing and Technology, vol. 3, pp. 22-24, 2004.

[17] J. Yao and Y. T. Zhang, "Bionic wavelet transform: a new timefrequency method based on an auditory model," IEEE Transactions on Biomedical Engineering, vol. 48, no. 8, pp. 856-863, 2001.

[18] J. Yao and Y. T. Zhang, "Cochlear is an inhomogeneous, active and nonlinear model," in Proceedings of the 1st Joint Meeting of BMES \& IEEE/EMBS, p. 1031, Atlanta, Ga, USA, October 1999.

[19] J. Yao and Y. T. Zhang, "From otoacoustic emission modeling to bionic wavelet transform," in Proceedings of the 22nd Annual International Conference of the IEEE Engineering in Medicine and Biology Society, vol. 1, pp. 314-316, Chicago, Ill, USA, July 2000.

[20] J. Yao and Y. T. Zhang, "The application of bionic wavelet transform to speech signal processing in cochlear implants using neural network simulations," IEEE Transactions on Biomedical Engineering, vol. 49, no. 11, pp. 1299-1309, 2002.

[21] X. Yuan, "Auditory model-based bionic wavelet transform for speech enhancement," M.S. thesis, Speech and Signal Processing Laboratory, Marquette University, Milwaukee, Wis, USA, 2003.

[22] D. L. Donoho and I. M. Johnstone, "Adapting to unknown smoothness via wavelet shrinkage," Journal of the American Statistical Association, vol. 90, no. 432, pp. 1200-1224, 1995.

[23] K. L. Park, K. J. Lee, and H. R. Yoon, "Application of a wavelet adaptive filter to minimise distortion of the ST-segment," Medical and Biological Engineering and Computing, vol. 36, no. 5, pp. 581-586, 1998.

[24] “The MIT-BIH Arrhythmia Database," http://physionet.ph. biu.ac.il/physiobank/database/mitdb/.

[25] P. E. McSharry, G. D. Clifford, L. Tarassenko, and L. A. Smith, "A dynamical model for generating synthetic electrocardiogram signals," IEEE Transactions on Biomedical Engineering, vol. 50, no. 3, pp. 289-294, 2003.
[26] P. E. McSharry and G. D. Clifford, "ECGSYN—a realistic ECG waveform generator," http://www.physionet.org/physiotools/ ecgsyn/.

Omid Sayadi was born in Shiraz, Iran, in 1983. He majored in biomedical engineering and received the B.S. degree from Shahed University of Tehran, Iran, in 2005. He was a Research Scientist with BehsazTeb Co., Tehran, from 2003 to 2005. He is currently an M.S. student of biomedical engineering at the Electrical Engineering Department of Sharif University of Technology, Tehran, Iran, and a member of Biomedical Signal and Image Processing Laboratory (BiSIPL). His research deals with dynamical models for ECG generation, modelbased ECG processing, the application of wavelet concepts, and especially multiadaptive bionic wavelet transform to biomedical signal processing solutions, signature verification, and efficient heart modeling.

Mohammad B. Shamsollahi was born in Qom, Iran, in 1965. He received the B.S. degree in electrical engineering from Tehran University, Tehran, Iran, in 1988, and the M.S. degree in electrical engineering, Telecommunications, from the Sharif University of Technology, Tehran, Iran, in 1991. $\mathrm{He}$ received the Ph.D. degree in electrical engineering, biomedical signal processing, from the University of Rennes 1, Rennes,

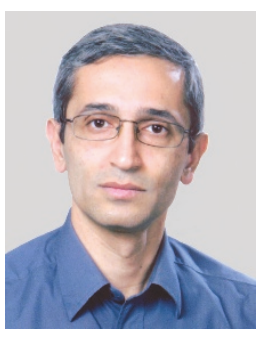
France, in 1997. Currently, he is an Assistant Professor with the Department of Electrical Engineering, Sharif University of Technology, Tehran, Iran. His research interests include biomedical signal processing, brain computer interface, time-scale and timefrequency signal processing. 